\title{
The increased expression of Piezo1 and Piezo2 ion channels in human and mouse bladder carcinoma
}

\author{
Ebru Önalan Etem ${ }^{1, A, D}$, Gülay Güleç Ceylan ${ }^{2, B, C, E, F}$, Seda Özaydın ${ }^{1, A-C}$, Cavit Ceylan ${ }^{3, A, E}$, Ibrahim Özercan ${ }^{4, A, B}$, Tuncay Kuloğlu u $^{5, A-C}$ \\ ${ }^{1}$ Department of Medical Biology, Faculty of Medicine, Firat University, Elazığ, Turkey \\ 2 Department of Medical Genetics, Faculty of Medicine, Yıldırım Beyazıt University, Ankara, Turkey \\ ${ }^{3}$ Urology Clinics, Yüksek Ihtisas Education and Training Hospital, Ankara, Turkey \\ ${ }^{4}$ Department of Pathology, Faculty of Medicine, Firat University, Elazığ, Turkey \\ ${ }^{5}$ Department of Histology and Embriology, Faculty of Medicine, Firat University, Elazığ, Turkey \\ A - research concept and design; B - collection and/or assembly of data; $\mathrm{C}$ - data analysis and interpretation; \\ $D$ - writing the article; $E$ - critical revision of the article; $F$ - final approval of the article
}

\section{Address for correspondence \\ Gülay Güleç Ceylan \\ E-mail: gulayceylan23@gmail.com}

Funding sources

None declared

Conflict of interest

None declared

Received on February 18, 2017

Reviewed on April 3, 2017

Accepted on May 7, 2017

\begin{abstract}
Background. Piezo1/2, a mechanically activated ion channel, is believed to play an important role in bladder carcinogenesis process. Piezo $1 / 2$ expression has not been previously reported in urinary bladder carcinoma, and little is known about its significance in bladder carcinogenesis.
\end{abstract}

Objectives. In our study, we aimed to evaluate the Piezo1 and Piezo2 expression as developmental in mouse bladder tissue and bladder cancer tissue of mice and humans.

Material and methods. The detection of developmental expression was performed on P0-P90 days in bladder tissue of Balb/c strain mice. Mice were divided into bladder cancer $(n=40)$ and control groups ( $n=10)$. Bladder cancer in mice was created by using $N$-butyl- $N$-(4-hydroxybutyl)nitrosamine (BBN). In the study, 60 human subjects were included, whose normal tissues were used as controls. After the histopathological evaluation, the expression of Piezo1/2 genes was examined by reverse transcription polymerase chain reaction (RT-PCR) and immunohistochemistry in tumor and normal tissues.

Results. In developmental period of the mice, Piezo7 expression increased on days 21 and 90, whereas Piezo2 expression increased on day 7 and decreased on day 90 in mouse bladder tissues. There was a significant increase in the expression of Piezo1/2 in both cancer groups compared to the control group. Piezo 1 expression was significantly increased at tumor size, stage and grade. Piezo2 expression was upregulated in high grade tumors in human subjects.

Conclusions. The developmental changes of Piezo expression on specific days demonstrate the role of these channels in bladder cancer development. The dysfunction of Piezo1/2 expression may contribute to the carcinogenesis of bladder cancer by causing proliferative changes and angiogenesis. The expression of Piezo1/2 can provide new prognostic information for disease progression.

Key words: bladder cancer, messenger RNA expression, Piezo channels

DOI

10.17219/acem/71080

Copyright

Copyright by Author(s)

This is an article distributed under the terms of the

Creative Commons Attribution Non-Commercial License

(http://creativecommons.org/licenses/by-nc-nd/4.0/) 


\section{Introduction}

Bladder cancer is the $4^{\text {th }}$ most common cancer type in men. ${ }^{1}$ Nearly $90 \%$ of patients with bladder cancer have transitional cell carcinoma of the bladder (TCC). Bladder cancers with a new diagnosis are usually - in $75 \%$ of cases - non-muscle invasive. ${ }^{2}$ According to several studies, there are many etiological factors that contribute to the disease, such as male gender, tobacco use, chemical agents, diet, exposure to cyclophosphamide, ionizing radiation, phenacetin-containing analgesics, and genetic factors. ${ }^{3,4}$

Some of the channel types localized in the plasma membrane and intracellular organelles are involved in carcinogenesis and contribute to the basic phenotypes, like metabolic reprogramming, migration, unlimited proliferative potential, apoptosis resistance, induction of angiogenesis, and invasion of the cancer cells. The channels that contribute to cancer development and progression have been defined as "oncogenic channels". ${ }^{5-7}$

Mechanotransduction is the conversion of a mechanical stimulus into chemical/biological activity. This is a basic physiological process of mammalian cells and it affects many critical processes, such as pain, embryonic development and vascular tone regulation. ${ }^{8}$ Mechanosensitive ion channels (MSCs) are associated with the physiological functions of some biological systems, e.g., the musculoskeletal system, neuromuscular system and/or respiratory system. ${ }^{9}$ Piezo1 and Piezo 2 are a unique class of a conserved family of nonselective cation channels that are activated by physical or mechanical stimuli. ${ }^{10-12}$ They have no sequence similarity with any other channel..$^{13}$ Both of these Piezo channels are cation-selective mechanical channels and they have a selectivity sequence of preference for ions $\mathrm{Ca}^{2+}>\mathrm{K}^{+}>\mathrm{Na}^{+}>\mathrm{Mg}^{2+} \cdot{ }^{24,15}$ Piezol and Piezo 2 are homologous and they were both cloned in 2010. ${ }^{8,13}$ Piezo proteins form a homotetramer of about 1.2 MDa and have been highlighted as a central pore module. ${ }^{13,16}$ Expression profiles of messenger RNA (mRNA) and proteins for Piezo1 and Piezo2 have been detected in numerous MSC tissues. ${ }^{10}$ The expression of Piezo1 has been shown in endothelial cells, red blood cells, cochlear hair cells, kidney, skin, lung, and bladder tissue. ${ }^{11,17,18}$ Piezo1 also plays a significant physiological role in the hematopoietic and cardiovascular system. ${ }^{11,12,19,20}$ It has been documented for its responsibility for cell volume regulation of red blood cells. Gain-of-function mutations of Piezo1 have been detected in cases with dehydrated hereditary stomatocytosis (DHS), which is characterized by a decreased volume of intracellular erythrocytes and a mild hemolysis. ${ }^{21-23}$

Homozygous mutant Piezo1 mice are embryonically lethal. ${ }^{11,19}$ However, blood cell-specific Piezo1 knockout mice display increased erythrocyte size and osmotic fragility. ${ }^{24}$ Piezo 2 has been shown to be expressed in the dorsal root and trigeminal ganglia, lung, bladder, and Merkel cells. ${ }^{17}$

The suggestion that Piezo1 and Piezo2 channels regulate mammalian development, physiology and carcinogenesis has yet to be clarified. Therefore, in this study, we aimed to evaluate Piezo1 and Piezo2 ion channel protein expressions in postnatal period (P0-P90) in mice; we also compared the expressions in human and mouse tumor and non-tumor bladder tissues.

\section{Material and methods}

\section{Patients}

We conducted a prospective controlled study with patients that were admitted with painless gross hematuria and low urinary tract symptoms (LUTS). The study was approved by the Firat University (Elazığ, Turkey) Local Ethics Committee and written informed consent was obtained from each participant before the study was initiated. Bladder tissue samples were obtained from 60 patients (14 females and 46 males). Transurethral resection was performed in the presence of bladder tumor and a biopsy was taken if the suspicion was determined. Routine pathological diagnoses were performed according to hematoxylin and eosin (H\&E) staining. During the pathological analysis, the samples were categorized according to the WHO/International Society of Urological Pathology (ISUP) 1998 classification. ${ }^{25}$ Patients in whom a pathological examination revealed TCC constituted the patient group and those with a benign pathology examination constituted the control. After a histopathological evaluation for urothelial carcinoma, the expression of Piezo1 and Piezo2 gene was analyzed by quantitative real-time polymerase chain reaction (qRT-PCR). Immunohistochemistry of the tumor and normal tissues of patients was performed to determine whether there was a difference between the expression of Piezo1 and Piezo2 channels in tumor and normal tissues.

\section{Animals}

The ethical Animal Research Committee of Firat University approved the study protocol. Balb/c-type mice were housed in standard plastic cages. Animals were maintained in light rooms for $12 \mathrm{~h}$ and in dark rooms for $12 \mathrm{~h}$. All animals were allowed free access to water and food. Bladder tissue samples of 2 mice for each day: P0, P7, P14, P21, P28, P36, and P90 were used in each postnatal period for determining the developmental expression. Mice were arranged as 2 groups: the bladder cancer group $(n=40)$ and the control group $(\mathrm{n}=10) . N$-butyl- $N$-(4-hydroxybutyl) nitrosamine (BBN) $(0.05 \%$ in the drinking water) was applied to induce bladder cancer in mice for 3 months. Carcinoma formation was expected for 4 months following this process. After the detection of pathologic carcinoma by a pathologist with $H \& E$ staining in 2 mice 4 months later, all animals were decapitated after $3 \%$ pentobarbital abdominal anesthesia. Bladder tissues were stored in liquid nitrogen at $-80^{\circ} \mathrm{C}$ for qRT-PCR and in $10 \%$ neutral buffered formalin for immunohistochemical analysis. 


\section{RNA extraction}

Total RNA was isolated from human and mouse bladder tissue using Trizol (Life Technologies, San Francisco, USA). Bladder tissue was homogenized using a homogenizer (Next Advance, Inc., Averill Park, USA). Total RNA was dissolved in $100 \mu \mathrm{L}$ of diethyl pyrocarbonate (DEPC)-treated $\mathrm{H}_{2} \mathrm{O}$, aliquoted and stored at $-80^{\circ} \mathrm{C}$. A Qubit ${ }^{\circledR} 2.0$ Fluorometer (Invitrogen by Life Technologies ${ }^{\mathrm{TM}}$, Zug, Austria) was used to determine RNA concentration and purity.

\section{Real-time polymerase chain reaction}

High Capacity cDNA Reverse Transcription Synthesis Kits (Applied Biosystems, Inc., Foster City, USA) were used for the conversion of total RNA to complementary DNA (cDNA), using $1 \mu \mathrm{g}$ of total RNA. Complementary DNA isolation was performed in a gradient thermal cycler (Biometra, Göttingen, Germany) with a profile of $25^{\circ} \mathrm{C}$ for $10 \mathrm{~min}$, $37^{\circ} \mathrm{C}$ for $120 \mathrm{~min}, 85^{\circ} \mathrm{C}$ for $5 \mathrm{~min}$, and $4^{\circ} \mathrm{C}$ for $60 \mathrm{~min}$.

\section{A}

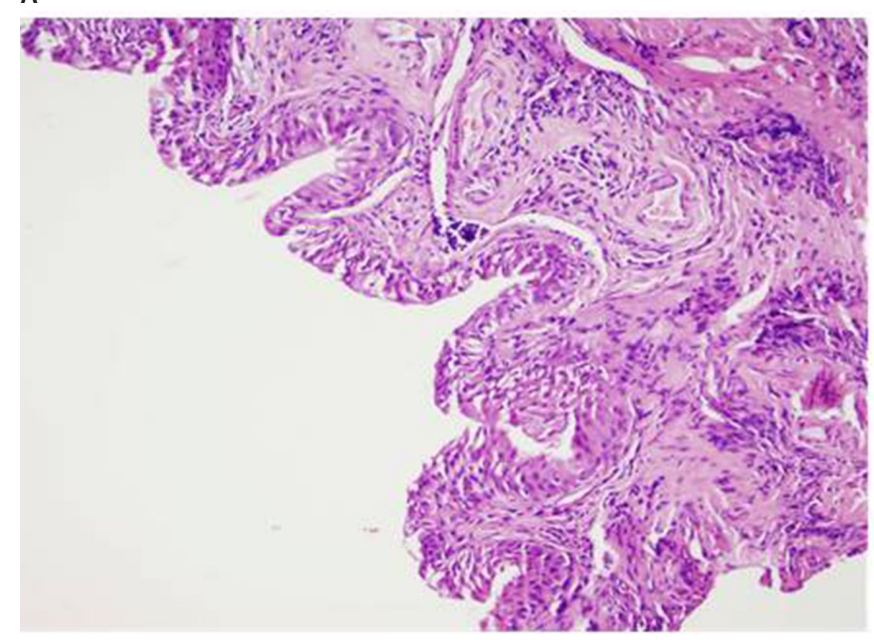

\section{B}

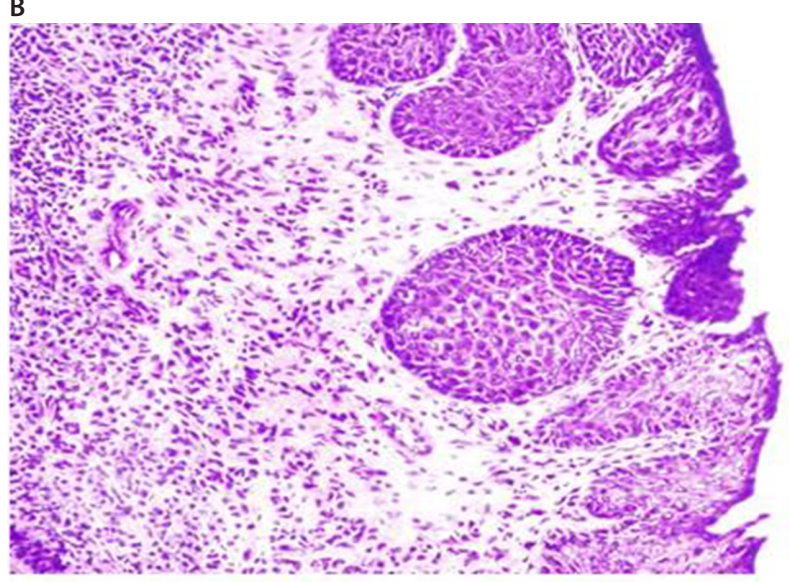

All of the samples were run in batch. As a negative control, samples were run simultaneously with no reverse transcriptase enzyme (RT negative) or RNA (no template controls) to control for RNA and genomic DNA contamination, respectively. ABI Prism 7500 Fast Real Time PCR Instrument (Applied Biosystems) was used for the reverse transcription polymerase chain reaction (RT-PCR) analysis using Piezo1 (Hs00207230-m1 and Mm01241549-m1) and Piezo2 (Hs00401026-m1 and Mm01265861-m1). All the results were standardized according to the levels of glyceraldehyde 3-phosphate dehydrogenase (GAPDH) (Hs02758991-g1 and Mm99999915-g1).

\section{Immunohistochemistry staining}

Human bladder sections (4-5 $\mu \mathrm{m}$ thick) were prepared from paraffin embedded blocks for immunohistochemical staining. Peptide blocking and immunohistochemical studies were performed with the indirect immunoperoxidase staining technique on paraffin-embedded material.

\section{C}

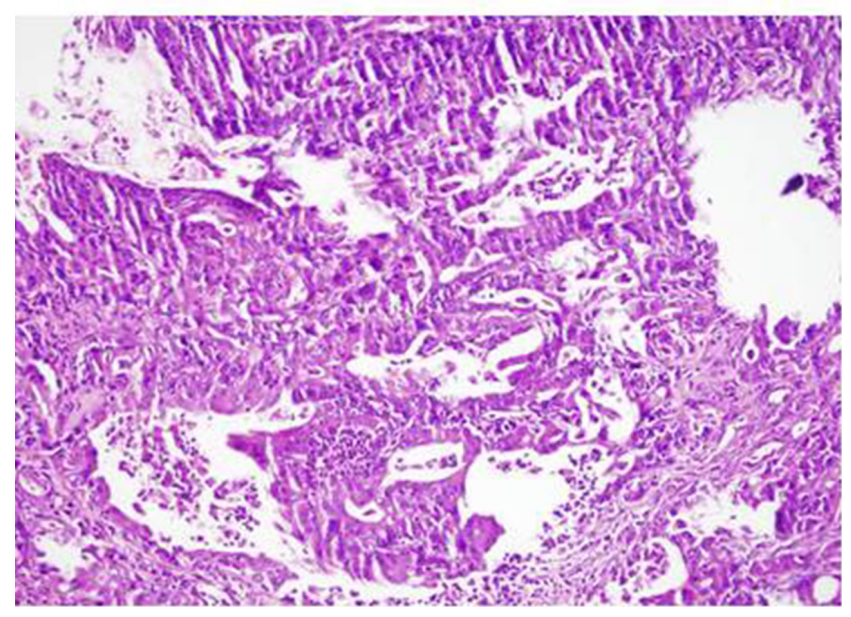

D

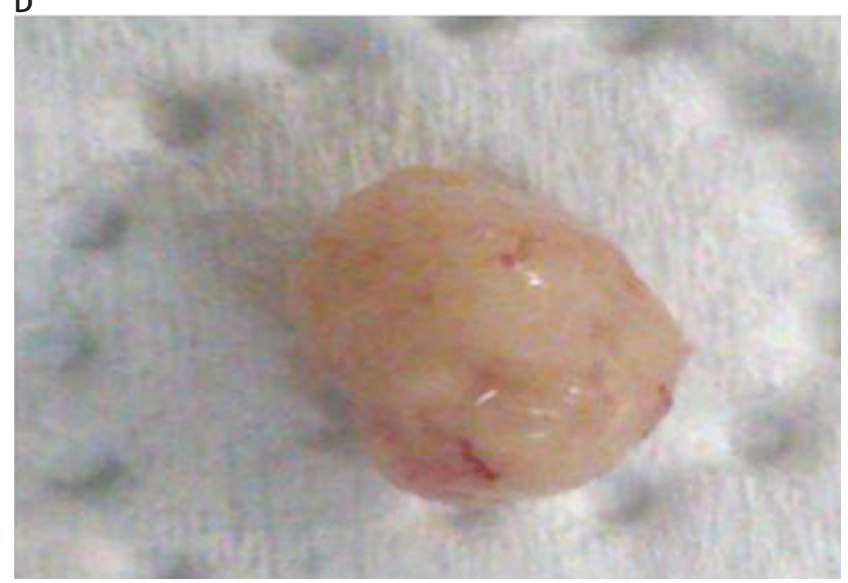

Fig. 1. Representative H\&E-stained images of urothelial cross-sections from BBN-induced bladder cancer in mice

Note the urothelial cancers in the BBN-induced mice: A - note the normal-appearing urothelium in the control group of mice; B - note the high-grade carcinoma-in-situ lesion in BBN-treated mice; C - note the high-grade, papillary bladder tumor in BBN-treated mice; magnification: $\times 400$; D - fresh tumor samples with adenocarcinomas in mice. 
Non-specific staining was blocked using a 20 min incubation with a serum-blocking solution (Immuno Cruz Staining System; Santa Cruz Biotechnology, Dallas, USA). The sections were incubated with a Piezo1 (NBP1-78537; Novus Biologicals, Littleton, USA) and Piezo2 (NBP1-78538; Novus Biologicals) antibody diluted 1:200 for $60 \mathrm{~min}$ in a humid chamber at room temperature. The sections were then incubated with streptavidin peroxidase (TS-125-HR; Lab Vision Corp., Fremont, USA) for $30 \mathrm{~min}$ after they were washed 3 times with phosphate buffered saline (PBS). Drops of 3-amino-9-ethylcarbazole (AEC) substrate + AEC chromogen (AEC Substrate, TA-015-HAS; AEC Chromogen, TA-002-HAC; Lab Vision Corp.) solution were applied, and the tissues were washed with PBS after labeling was checked by light microscopy. Mayer's hematoxylin was used for the counterstaining of sections, rinsed in PBS and distilled water, and then mounted with a closure solution (Large Volume Vision Mount, TA-125-UG; Lab Vision Corp.). After the slides were analyzed by the BX50 optical microscopy (Olympus Corp., Tokyo, Japan), they were photographed. The evaluation of immunohistochemical staining was performed semi-quantitatively, based on the spreading and levels of the staining. The levels for immunostaining in bladder tissue were detected by subjective visual scoring of the brown stain. Then, they were compared with normal tissue. The scoring levels were as follows: weak staining intensity (nearly equal to normal tissue $=0$ ), moderate staining intensity (1) and strong staining intensity (2).

\section{Statistical analysis}

Statistical Package for the Social Sciences (SPSS) software v. 22 for Windows (IBM, Armonk, USA) was used for the analysis of data for each experiment. If the data satisfied the suppositions of normality and homogeneity of parametric analyses (one-way independent measure ANOVA and t-tests), variance was performed along with Tukey's honest significant difference (HSD) posthoc test, if it was appropriate. A p-value of $<0.05$ was accepted as statistically significant. All data was presented as mean \pm standard deviation (SD) or standard error of the mean (SEM).

A

Developmental Piezo1 and Piezo2 mRNA expression in mouse bladder
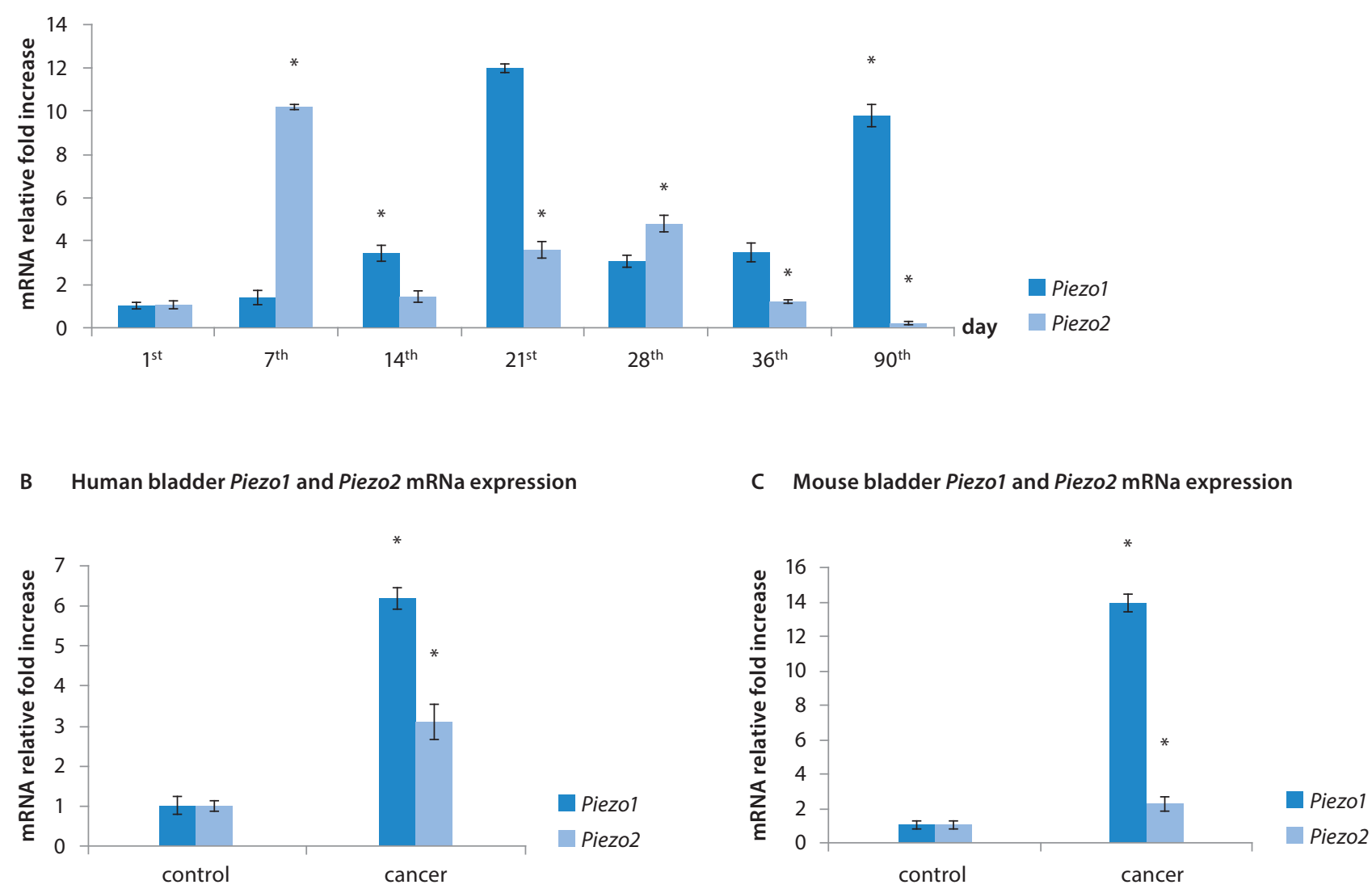

Fig. 2. Quantitative RT-PCR analysis of Piezo1 and Piezo2 in bladder tissue in mice and humans

A - results are expressed as mean \pm SD for 14 mice (2 mice for each day) on day PO, P7, P14, P21, P28, P36, and P90 for determining the developmental expression; B results are expressed as mean \pm SD for 10 mice from the control group and $31 \mathrm{BBN}$-treated animals; $\mathrm{C}$ - results are expressed as mean \pm SD for cancerous and healthy tissue of 60 patients; error bars represent SD significantly different $(p<0.05)$ from control or PO ; * shows the statistical significance. 


\section{Results}

\section{Molecular findings}

A total of 60 patients with urothelial carcinoma were included in the analysis for staging. The majority (63.3\%) of the urothelial tumors were in the T1 stage. All patients in the study group were smokers. Patient characteristics are shown in Table 1.

In the BBN-treated group, 9 animals died during the procedure. A histopathologic evaluation of all the bladders was used to determine $\mathrm{BBN}$-induced carcinogenesis and revealed benign proliferative changes in 32.2\% (10/31) and urothelial carcinoma in $67.7 \%(21 / 31)$ of the animals treated with BBN (Fig. 1). In situ, carcinoma in 66.6\% (14/21) samples and adenocarcinoma in $33.3 \%$ (7/21) samples of urothelial carcinomas was also determined. As expected, this contrasted significantly with the control group, in which no evidence of bladder cancer was detected (Fig. 1).

We first performed the gene expression analysis by RT-PCR in tumor samples. Piezo1 expression significantly increased on day P14, P21, P28, P36, and P90 in comparison with day $\mathrm{P} 1$, whereas Piezo2 expression significantly increased on day P7, P21 and P28, and decreased on day P90 compared to day P1 (Fig. 2A). Piezo1 was detected to be upregulated in $53.3 \%$ (32/60), downregulated in $13.33 \%(8 / 60)$ and not changed in $30.7 \%(20 / 60)$ of the human bladder tissue samples. Piezo 2 was detected to be upregulated in $60 \%$ (36/60), downregulated in $20 \%$ $(12 / 60)$ and not changed in $20 \%(12 / 60)$ of the human bladder tissue samples. The expression levels of Piezo1 and Piezo2 calculated in all the human bladder cancer group tissues was significantly increased compared to normal tissue (t-test; $\mathrm{p}=0.00$ and $\mathrm{p}=0.00$, respectively) (Fig. 2B). On the other hand, the expression levels of Piezo1 and Piezo2 in the BBN-induced bladder cancer group was significantly higher than in the normal control group (t-test; $\mathrm{p}<0.00$ and $\mathrm{p}=0.04$, respectively) (Fig. $2 \mathrm{C}$ ).

The detection rate of Piezo1 was $100 \%$ in bladder carcinoma specimens. The expression of Piezo1 in bladder carcinoma was significantly associated with tumor size and stage, but it had no association with other clinicopathological features. The expression of Piezo1 in bladder carcinoma patients with $\geq 3$ tumor size was
A

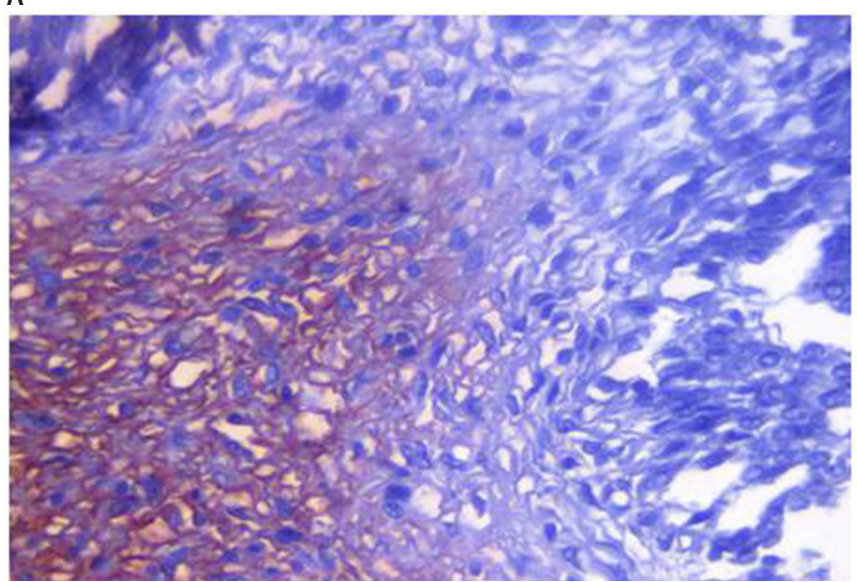

B

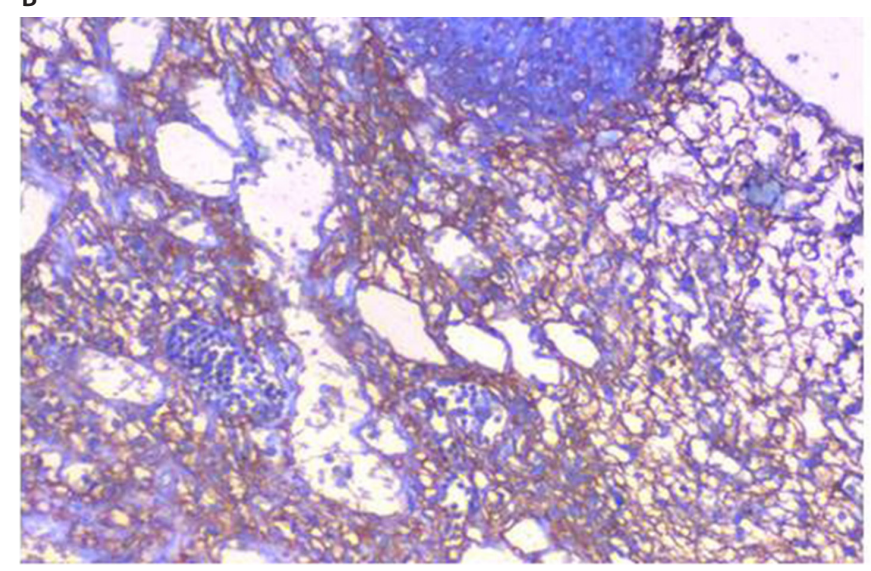

C

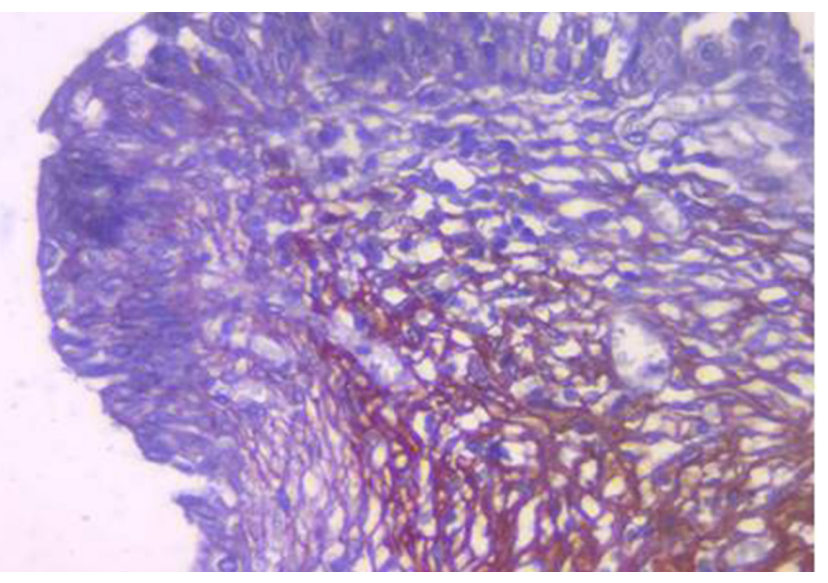

D

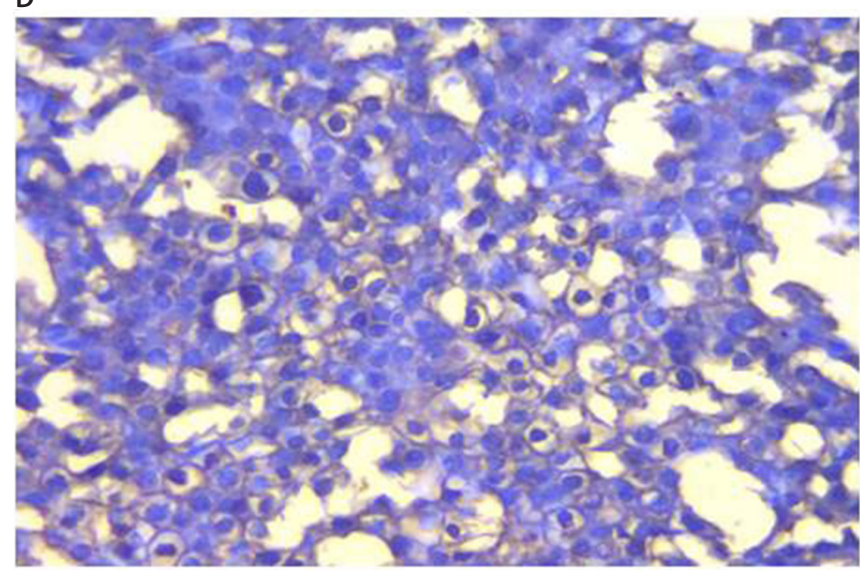

Fig. 3. Immunohistochemical staining for Piezol and Piezo2 in human bladder tissue

Representative bladder sections stained for Piezol (A - control; B - cancer) and Piezo2 (C - control; D - cancer) in carcinomas and control tissue in human bladder samples. Piezol and Piezo2 staining was detected especially in the cytoplasm and membrane urothelial cells. Note the marked upregulation of both Piezol and Piezo2 in human bladder cancer. Scale bar: $20 \mu \mathrm{m}$; magnification $\times 200$. 
significantly higher than in bladder carcinoma patients with $<3$ tumor size $(\mathrm{p}=0.00)$. Piezo1 expression was significantly upregulated in $\geq \mathrm{pT} 2$ (muscle invasive bladder cancer with pathological findings) as compared to pT0 (no evidence of residual carcinoma in the cystectomy specimens after an initial cancer diagnosis in the biopsy or transurethral resection specimens) and pT1 (high-risk non-muscle invasive bladder cancer with pathological findings) patients (pT0 vs $\geq \mathrm{pT} 2, \mathrm{p}=0.006$; $\mathrm{pT} 1 \mathrm{vs} \geq \mathrm{pT} 2$, $\mathrm{p}=0.000$, respectively). Piezo2 expression was increased in $\geq \mathrm{pT} 2$ patients as compared to pT0 patients (pT0 vs $\geq \mathrm{pT} 2, \mathrm{p}=0.003)$. pT0 vs $\mathrm{pT} 1$ and $\mathrm{pT} 1$ vs $\geq \mathrm{pT} 2$ were not significantly different ( $\mathrm{p}>0.05)$. A higher expression level of Piezo2 in high-grade tumors was detected ( $\mathrm{p}=0.003$ ). The data also demonstrated that the expression level of Piezo2 had no significant association with tumor grade. Piezo1 and Piezo2 expression was not significantly different in bladder cancer specimens with lymph node metastasis as compared to specimens without lymph node metastasis; there were also no differences with regard to age and gender ( $\mathrm{p}>0.05)$.

\section{Immunohistochemical findings}

To examine the localization of Piezo1 and Piezo2 in the human bladder, the immunohistochemical analysis was performed. Immunohistochemistry revealed that Piezo1 immunoreactivity was mainly observed in the urothelial layer with less immunoreactivity in stromal tissues (Fig. 3A,3B). In contrast, little Piezo2 expression was detected in urothelial tissues (Fig. 3C,3D). Piezo1 and Piezo2 were mainly localized in the plasma membrane and the cytoplasm near the nucleus.

\section{Discussion}

In the present study, we investigated pathological changes in Piezo1 and Piezo2 in the bladder cancers of humans and mice. We obtained the following novel findings:

- mRNA expressions of Piezo1 and Piezo2 genes in cancerous bladder tissues were increased as compared to control bladder tissues;

- Piezo1 expression was correlated with tumor stage, grade and size;

- Piezo2 expression increased significantly only in $\geq \mathrm{pT} 2$ patients as compared to pT0.

Piezo1 and Piezo2 proteins take part in mechanosensory processes and they are stretch-activated cation channels. Almost every cell can sense the mechanical signals. In particular, stretch-activated cation channels in cells have a basic role in sensing these mechanical signals. ${ }^{8,26,27}$ Coste et al. found that the mRNA expression of Piezo1 is the highest in the bladder, skin and lungs of adult mice. ${ }^{10}$ The loss or absence of Piezol plays an important role in increased cell migration and metastases of lung cancer.
Table 1. Distribution of variables in bladder cancer patients

\begin{tabular}{|c|c|}
\multicolumn{1}{|c|}{ Characteristics } & Values \\
\hline Age [years], mean \pm SD (range) & \\
male & $64.9 \pm 4.29(56-76)$ \\
female & $62.2 \pm 3.51(58-70)$ \\
Gender, $n$ (\%) & \\
male & $46(76.6 \%)$ \\
female & $14(23.3 \%)$ \\
\hline Tumor size & \\
$<3$ & 34 \\
$\geq 3$ & 26 \\
Lymph node involvement & \\
no & 57 \\
yes & 3 \\
\hline Tumor grade & \\
low & 48 \\
high & 12 \\
\hline Tumor stage & \\
pT0 & 8 \\
pT1 & \\
$\geq$ pT2 & 38 \\
\hline Total & 14 \\
\hline & 60 \\
\hline
\end{tabular}

A decrease of Piezo1 in gastric tumors has been related to reduced cell migration. ${ }^{14,17,28}$ The authors also described the extrusion of living cells at colon surfaces. This suggests that the loss of Piezo1 in colon tissue could lead to polyp formation. ${ }^{17}$ All of these reports point at Piezo proteins as critical for cell migration, elongation and proliferation. In the present study, we observed similar results; mRNA expression was high in bladder cancer tissues, but higher expression levels could lead to cell migration. To support our results, the knockdown of Piezo1 in gastric epithelium cancer cells was shown to reduce cell migration. The authors suggested that Piezol overexpression promotes the invasion and metastasis of gastric cancer. ${ }^{17}$ Increases in the Piezo ion channel expression can cause dysfunction and leakage at these ion channels. This process may also result in proliferative changes in bladder cancer tissue. ${ }^{29}$

According to a recent study, haploinsufficiency of Piezo1 leads to endothelial abnormality, indicating the importance of this gene for vascular structure. ${ }^{19}$ Angiogenesis is a finely tuned process, which is the result of the equilibrium between pro- and anti-angiogenic factors. In solid tumor angiogenesis, the balance is highly in favor of the production of new, but poorly functioning blood vessels, which are initially intended to provide growing tumors with nutrients and oxygen. ${ }^{30}$ Piezo1 ion channels may thus affect different hallmarks of cancer, especially tumor angiogenesis.

Yang et al. reported that Piezo2 knockdown decreased angiogenesis and vascular hyperpermeability, and inhibited endothelial cell proliferation, migration and tube formation. Piezo 2 knockdown in endothelial cells resulted in the suppression of cell proliferation, migration and invasion of glioma tumor cells, which regulate glioma angiogenesis via $\mathrm{Ca}^{2+} / \mathrm{Wnt} 11 / \beta$-catenin signaling. ${ }^{26}$ These studies 
provide evidence for Piezo2 as a critical regulator of tumor angiogenesis and vascular permeability. The present study showed that Piezo2 expression was increased in bladder tumor samples compared to human and mouse control tissues. Inspired by these findings, we speculated that Piezo 2 may contribute to the carcinogenesis of bladder cancer by regulating tumor angiogenesis. However, the function of Piezo2 in bladder tumor angiogenesis has not been elucidated.

It is not known whether the changes in Piezo channel expression in bladder cancer tissue occur due to the basic steps of cancer progression or secondary to other variations. For this reason, we aimed to investigate postnatal expressions of Piezo channels in this study. Expression variation on certain days supports the notion that these channels may play a role in bladder development.

\section{Conclusions}

According to our study, Piezo proteins are thought to be involved in cancer and they regulate mechanical transmission. Increased expression of Piezo1 and Piezo2 in bladder cancer indicates that these channels may be therapeutic targets. The use of blockers for these channels has been determined to inhibit the cell proliferation. Further research will reveal whether Piezo1 and Piezo2 are causally involved in the carcinogenesis and progression of bladder cancer, which represents a potential therapeutic target or a prognostic factor.

\section{References}

1. Silverman DT, Devesa SS, Moore LE, Rothman N. Bladder cancer. In: Schottenfeld D, Fraumeni J, eds. Cancer Epidemiology and Prevention. $3^{\text {rd }}$ ed. New York, NY: Oxford University Press; 2006:156-160.

2. Siegel R, Ma J, Zou Z, Jemal A. Cancer statistics. CA Cancer J Clin. 2014;64:9-29.

3. Heney NM, Ahmed S, Flanagan MJ, et al. Superficial bladder cancer: Progression and recurrence. J Urol. 1983;130:1083-1086.

4. Vlaovic P, Jewett MA. Cyclophosphamide-induced bladder cancer. Can JUrol. 1999;6:745-748.

5. Leanza L, Biasutto L, Managò A, Gulbins E, Zoratti M, Szabò I. Intracellular ion channels and cancer. Front Physiol. 2013;4:227.

6. Hoffmann EK, Lambert IH. Ion channels and transporters in the development of drug resistance in cancer cells. Philos Trans $R$ Soc Lond $B$ Biol Sci. 2014;369(1638):20130109.

7. Munaron L. Systems biology of ion channels and transporters in tumor angiogenesis: An omics view. Biochim Biophys Acta. 2015;1848(10 Pt B):2647-2656.

8. Xiao R, Xu XZ. Mechanosensitive channels: In touch with Piezo. Curr Biol. 2010;20:R936-R938.

9. Coste B, Houge G, Murray MF, et al. Gain-of-function mutations in the mechanically activated ion channel PIEZO2 cause a subtype of distal arthrogryposis. Proc Natl Acad SciU S A. 2013;110:4667-4672.

10. Coste B, Mathur J, Schmidt M, et al. Piezo1 and Piezo2 are essential components of distinct mechanically activated cation channels. Science. 2010;330:55-60.

11. Ranade SS, Woo SH, Dubin AE, et al. Piezo2 is the major transducer of mechanical forces for touch sensation in mice. Nature. 2014;516:121-125.

12. Woo SH, Ranade $S$, Weyer $A D$, et al. Piezo2 is required for Merkel-cell mechanotransduction. Nature. 2014;509:622-626.

13. Coste B, Xiao B, Santos JS, et al. Piezo proteins are pore-forming subunits of mechanically activated channels. Nature. 2012;483:176-181.
14. Gottlieb PA, Bae C, Sachs F. Gating the mechanical channel Piezo1: A comparison between whole-cell and patch recording. Channels (Austin). 2012;6:282-289.

15. Soattin L, Fiore M, Gavazzo P, et al. The biophysics of Piezo1 and Piezo2 mechanosensitive channels. Biophys Chem. 2015;208:26-33.

16. Ge J, Li W, Zhao Q, et al. Architecture of the mammalian mechanosensitive Piezo1 channel. Nature. 2015;527:64-69.

17. Yang XN, Lu YP, Liu J, et al. Piezo1 is as a novel trefoil factor family 1 binding protein that promotes gastric cancer cell mobility in vitro. Dig Dis Sci. 2014;59:1428-1435.

18. Cahalan SM, Lukacs V, Ranade SS, Chien S, Bandell M, Patapoutian A. Piezo1 links mechanical forces to red blood cell volume. Elife. 2015;22:4. doi: 10.7554/eLife.07370

19. Bae C, Gnanasambandam R, Nicolai C, Sachs F, Gottlieb PA. Xerocytosis is caused by mutations that alter the kinetics of the mechanosensitive channel PIEZO1. Proc Natl Acad SciUSA. 2013;110:E1162-1168.

20. Li J, Hou B, Tumova S, et al. Piezo1 integration of vascular architecture with physiological force. Nature. 2014;515:279-282.

21. Faucherre A, Kissa K, Nargeot J, Mangoni ME, Jopling C. Piezo1 plays a role in erythrocyte volume homeostasis. Haematologica. 2014;99:70-75.

22. Albuisson J, Murthy SE, Bandell M, et al. Dehydrated hereditary stomatocytosis linked to gain-of-function mutations in mechanically activated PIEZO1 ion channels. Nat Commun. 2013;4:1884.

23. Andolfo I, Alper SL, De Franceschi L, et al. Multiple clinical forms of dehydrated hereditary stomatocytosis arise from mutations in PIEZO1. Blood. 2013;121:3925-3935.

24. Demolombe S, Duprat F, Honoré E, Patel A. Slower Piezo1 inactivation in dehydrated hereditary stomatocytosis (xerocytosis). Biophys J. 2013;105(4):833-834.

25. Epstein Jl, Amin MB, Reuter VR, Mostofi FK. The World Health Organization/International Society of Urological Pathology Consensus Classification for urothelial (transitional cell) neoplasms of the urinary bladder. Am J Surg Pathol. 1998;22:1435-1438.

26. Yang H, Liu C, Zhou RM, et al. Piezo2 protein: A novel regulator of tumor angiogenesis and hyperpermeability. Oncotarget. 2016;7:44630-44643.

27. Zarychanski R, Schulz VP, Houston BL, et al. Mutations in the mechanotransduction protein PIEZO1 are associated with hereditary xerocytosis. Blood. 2012;120(9):1908-1915.

28. McHugh BJ, Murdoch A, Haslett C, Sethi T. Loss of the integrin-activating transmembrane protein Fam38A (Piezo1) promotes a switch to a reduced integrin-dependent mode of cell migration. PLOSONE. 2012;7:e40346.

29. Li C, Rezania S, Kammerer S, et al. Piezo1 forms mechanosensitive ion channels in the human MCF-7 breast cancer cell line. Sci Rep. 2015;5:8364.

30. Martins JR, Penton D, Peyronnet R, et al. Piezo1-dependent regulation of urinary osmolarity. Pflugers Arch. 2016;468:1197-1206. 